\title{
Enhancement of Tryptic Peptide Signals from Tissue Sections using MALDI IMS Post-ionization (MALDI-2)
}

Josiah C. McMillen ${ }^{1,2}$, Danielle B. Gutierrez ${ }^{2,3}$, Audra M. Judd ${ }^{2}$, Jeffrey M. Spraggins ${ }^{1-3^{*}}$, and Richard M. Caprioli ${ }^{1-5}$.

${ }^{1}$ Department of Chemistry, Vanderbilt University, 7330 Stevenson Center, Station B 351822, Nashville, TN 37235, USA

${ }^{2}$ Mass Spectrometry Research Center, Vanderbilt University, $46521^{\text {st }}$ Ave S \#9160, Nashville, TN 37235, USA

${ }^{3}$ Department of Biochemistry, Vanderbilt University, 607 Light Hall, Nashville, TN 37205, USA

${ }^{4}$ Department of Pharmacology, Vanderbilt University, 2220 Pierce Avenue, Nashville, TN 37232, USA

${ }^{5}$ Department of Medicine, Vanderbilt University, $116121^{\text {st }}$ Ave S, Nashville, TN 37232, USA

*Corresponding Author: Jeffrey M. Spraggins, Jeff.Spraggins@vanderbilt.edu

KEYWORDS. Imaging mass spectrometry, Matrix-assisted laser desorption/ionization, MALDI-2, post-ionization, peptides

\begin{abstract}
Matrix-assisted laser desorption/ionization imaging mass spectrometry (MALDI IMS) allows for highly multiplexed, unlabeled mapping of analytes from tissue sections. However, further work is needed to improve sensitivity and depth of coverage for protein and peptide IMS. We demonstrate signal enhancement of proteolytic peptides from thin tissue sections of human kidney by conventional MALDI (MALDI-1) augmented using a second ionizing laser (termed MALDI-2). Proteins were digested in situ using trypsin prior to IMS analysis. For tentative identification of peptides and proteins, a tissue homogenate from the same tissue analyzed by IMS was analyzed by LC-MS/MS. These proteins were digested in silico to generate a database of theoretical peptides to then match to MALDI IMS datasets. Peptides were tentatively identified by matching the MALDI peak list to the database peptide list employing a 5 ppm error window. This resulted in $314 \pm 45(n=3)$ peptides and 1,112 $\pm 84(n=3)$ peptides for MALDI-1 and MALDI-2, respectively. Protein identifications requiring two or more peptides per protein resulted in $55 \pm 13$ proteins with MALDI-1 and $205 \pm 10$ with MALDI-2. These results demonstrate that MALDI-2 provides enhanced sensitivity for the spatial mapping of tryptic peptides and significantly increases the number of proteins identified in IMS experiments.
\end{abstract}

The spatial mapping of peptides and proteins is crucial for understanding the underlying molecular drivers of tissue biology and pathology. ${ }^{1,2}$ Protein distributions in tissue are highly dependent on expression within cell types and on molecular changes in the tissue microenvironment. ${ }^{3,4}$ Generally, peptides and proteins are identified by mass spectrometry technologies from bulk tissue homogenates, but spatial information is not typically retained with these methods. In contrast, imaging of tissue using immunohistochemistry allows for sensitive analyte detection and high spatial resolution mapping, but requires a priori knowledge of the analyte of interest and is limited in the number of targets that can be imaged in a single experiment. Matrix-assisted laser desorption/ionization imaging mass spectrometry (MALDI IMS) of peptides and proteins combines the advantages of both MS and immunohistochemistry by providing high sensitivity and specificity analyte detection as well as untargeted, multiplexed mapping of hundreds-to-thousands of peptides and proteins from a single experiment.

Protein IMS has been reported for the intact detection of large proteins up to $200 \mathrm{kDa}^{5}$ but the practical upper mass limit is $\sim 50 \mathrm{kDa} .{ }^{6}$ High performance mass analyzers (e.g. FT-ICR and Orbitrap) provide superior mass resolving power and accuracy but have limited ion transmission efficiency at high $\mathrm{m} / \mathrm{z}$ and analyses are typically limited to $<30 \mathrm{kDa}$ for protein IMS experiments. ${ }^{7,8}$ To access larger proteins, a bottom-up approach is employed by applying a proteolytic enzyme such as trypsin to the tissue surface and imaging the resulting peptides. ${ }^{9-11}$ Using mass accuracy, multiple peptides with similar localization that match to a single protein derive a preliminary identification for the proteins. ${ }^{9,10}$
Although peptide and protein IMS workflows are routine, proteomic coverage and sensitivity remain as challenges. Attempts to increase proteomic coverage include using multiple, complementary enzymes for in situ digestion. ${ }^{12}$ Strategies to improve sensitivity through sample preparation approaches have included development of novel MALDI matrices ${ }^{13}$ and tissue washes to remove interfering lipid and metabolite species that suppress peptide signals. ${ }^{14}$ Other approaches include denaturing proteins using methods such as antigen retrieval to allow trypsin to access more sites for digestion. ${ }^{11} \mathrm{Di}$ gestion condition variables such as time, temperature, and humidity have also been optimized to improve digestion efficiency while maintaining peptide localization within a tissue section. ${ }^{15,16}$ Nevertheless, increased sensitivity is needed to improve proteomic coverage for bottom-up IMS experiments.

Many instrumental technologies can be employed to improve sensitivity for IMS experiments. ${ }^{17}$ One technology that has been shown to dramatically increase signal for MALDI IMS for some analytes is laser-based post-ionization. ${ }^{18,19}$ This approach ionizes some of the abundant neutrals that are generated during the initial MALDI event. Post-ionization by MALDI- 2 employs a secondary laser positioned parallel to, and above the sample to irradiate the plume generated by the initial MALDI event thereby increasing the fraction of molecules that are ionized. MALDI- 2 has been shown to increase ion intensity up to 100 -fold for lipids and thereby increase the number of identified species by a factor of two. MALDI- 2 postionization has been demonstrated to increase intensity for many 
classes of biologically relevant analytes including lipids, ${ }^{18-20}$ saccharides ${ }^{18}$ liposoluble vitamins, ${ }^{18} \mathrm{~N}$-linked glycans, ${ }^{21}$ certain pharmaceutical compounds, ${ }^{20}$ and some protein complexes. ${ }^{22}$ However there have been no reports of intensity gains using MALDI-2 postionization of peptides. Here, we demonstrate the use of MALDI-2 post-ionization for the enhancement of tryptic peptides from human kidney tissue sections.

To evaluate the feasibility of using MALDI-2 for IMS of tryptic peptides, sections of fresh frozen human kidney were prepared in technical triplicate. Human kidney tissues were collected as part of normal non-neoplastic portions of nephrectomy samples and were prepared for peptide analysis as described in the supplemental file. The MALDI matrix ( $\alpha$-cyano-4-hydroxy-cinnamic acid) was applied by pneumatic sprayer (HTX Technologies). Tissue sections were analyzed in positive ion mode using an Orbitrap Elite instrument equipped with a MALDI ion source (Spectroglyph), which included a second laser for MALDI-2 functionality ( $266 \mathrm{~nm}$, CryLaS) as described previously. ${ }^{23}$ Samples were analyzed at $50 \mu \mathrm{m}$ spatial resolution with MALDI-1 and MALDI-2. Similar and adjacent regions of the same section were analyzed, and the ion images contained a $\sim 15000$ in each region. Averaged spectra were deisotoped and peak picked based on a signal-to-noise ratio $(\mathrm{S} / \mathrm{N})$ threshold of 10. A comparison of representative averaged mass spectra generated from both analysis modes is displayed in Figure 1. Analysis with MALDI-1 resulted in 515 peaks whereas MALDI-2 allowed for detection of 1443 peaks. The overall spectral intensity was greater with MALDI-2 $\left(2.6 \times 10^{3}\right)$ as compared to MALDI-1 $\left(8.0 \times 10^{2}\right)$. These data indicate that there are significant signal and molecular coverage improvements for peptide IMS experiments with MALDI-2.

The human kidney tissue sections were imaged optically using autofluorescence (Figure $2 \mathbf{A}$ ), and the sections contain the major functional units of the kidney including the cortex, medulla, and glomeruli. Highlighted ion images were selected to provide examples of peptides that localize to these regions and throughout the tissue (Figure 2 B-E). Tentative peptide identification from IMS data was performed in three steps. First, a tissue homogenate of the same tissue used for IMS was analyzed using bottom-up LC-MS/MS proteomic protocols ${ }^{24,25}$ to identify proteins. Next, these proteins were digested in silico using Protein Prospector to obtain a database of all possible tryptic peptides. Finally, deisotoped IMS peak lists from MALDI-1 and MALDI-2 analyses (with S/N of 10 or greater) were compared to the peptide data from the in silico digestion. While this approach could potentially increase the number of false positives for proteins, it allows for annotation of possible peptides detected by MALDI-1 and MALDI-2 that may have gone undetected during LC-MS/MS analysis.

The theoretical $\mathrm{m} / z$ values of protonated peptides from the in silico digestion were matched to peak lists from the average IMS mass spectra with a mass tolerance of $5 \mathrm{ppm}$. Tentative protein identification was completed by peptide mass fingerprinting wherein the detection of two or more peptides corresponding to the same protein was used for protein identification. ${ }^{15,26}$ To deal with multiple peptide matches to a single MALDI ion, a simple scoring system was implemented that 1) ranked proteins in the LC-MS/MS dataset using sequence coverage and the number of identified peptides per protein normalized to molecular weight, and 2) ranked the
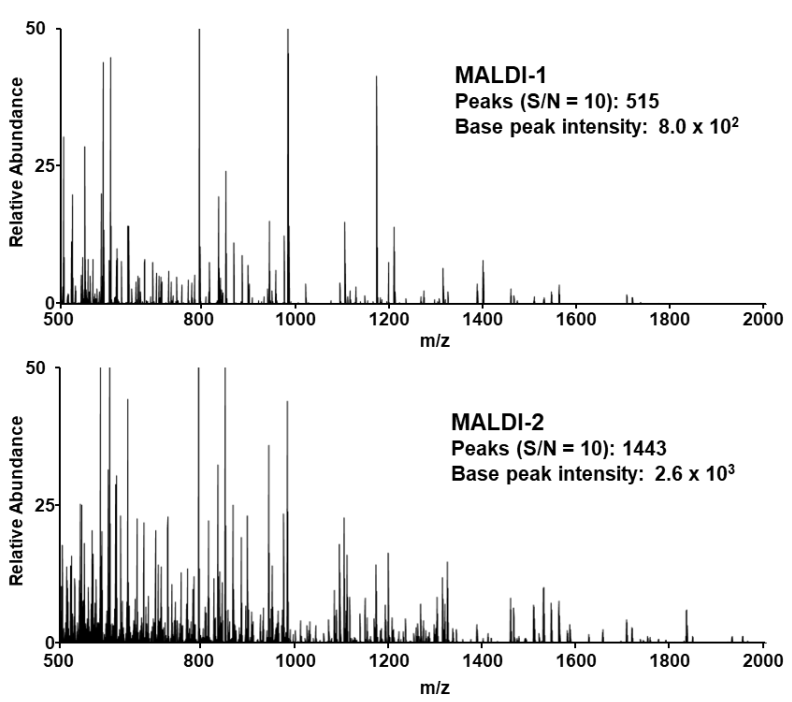

Figure 1. Average mass spectra of peptide ion images with (A) MALDI-1 and (B) MALDI-2 from IMS of human kidney sections digested in situ. Intensity of spectra is scaled to $50 \%$ relative abundance to highlight svectral differences.

mass accuracy of the MALDI-to-peptide matches as well as the number of potential peptides per protein for each match. The scoring system also considered the number of potential matches with the same mass difference. This identification strategy needs further validation as it is possibly biased toward higher molecular weight proteins. It was implemented here to provide tentative IDs to further compare MALDI and MALDI-2.

Many peptides not detected with MALDI-1 alone were detected with MALDI-2 (Figure 2 C-F), and MALDI-2 provided improved sensitivity for many ions already detectable with MALDI1 (Figure 2 B). Complementary ion images for the identified proteins in Figure 2 are shown in Figures S1-S5. Further, MALDI-2 provided for enhanced signal for $99 \%$ of identified peptides (986/988) from a representative sample. Two peptides decreased in signal with MALDI- 2 which may be caused by matrix adduct formation or selective photoionization as proposed by others for lipids. ${ }^{27}$ To determine if certain amino acids or molecular characteristics affect MALDI-2 of peptides, amino acid composition analysis was performed (Figure S7). Significant differences in amino acid composition were discovered but no correlation in specific amino acid characteristics such as acidity/basicity, polarity, or charge was found. Additionally, peptide hydropathy values for each peptide were calculated based on peptide grand averages of hydropathy using amino acid hydropathy values from Kyte and Doolittle ${ }^{28,29}$ but no 


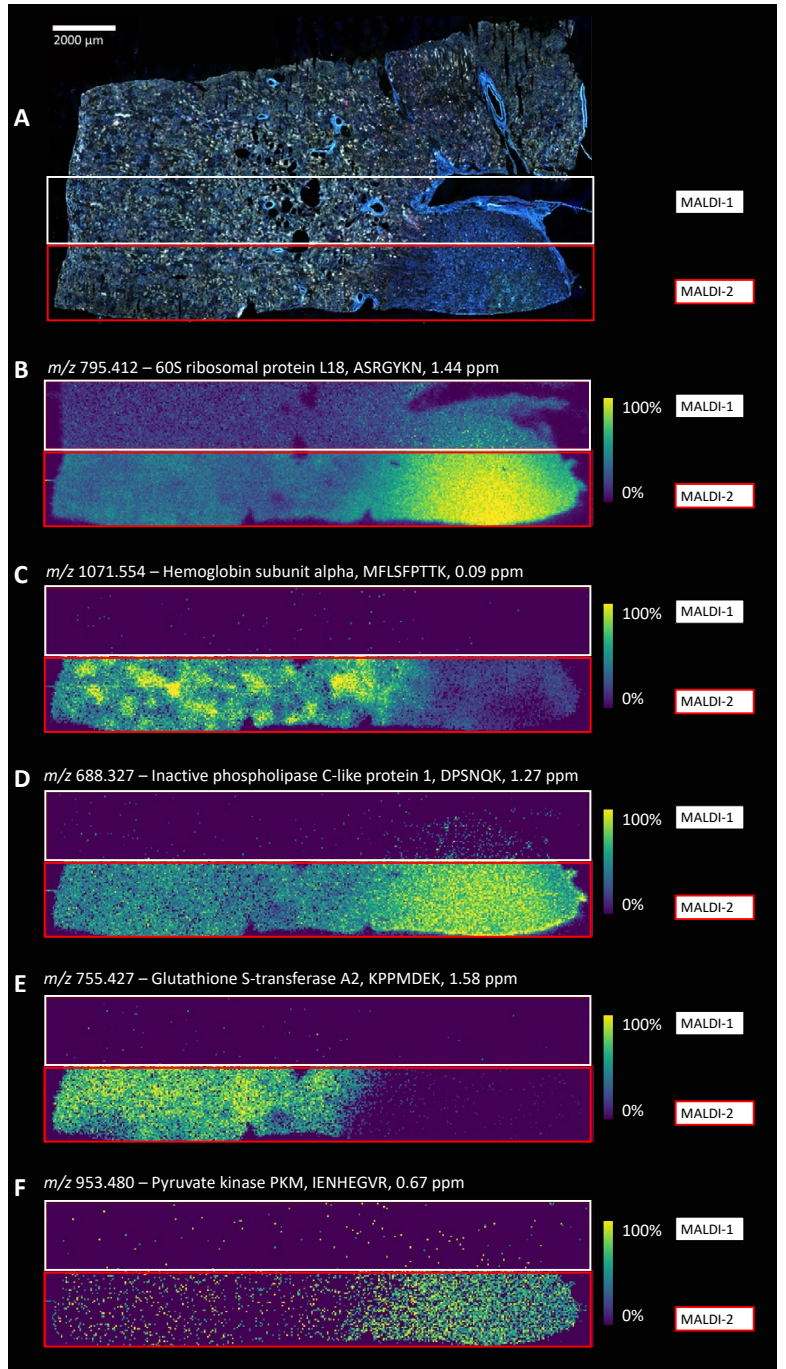

Figure 2. Autofluorescence image of human kidney (A) and ion images of peptides that localize to different tissue functional units of the kidney. Ion images were obtained via MALDI-1 (white outline, above) and MALDI-2 (red outline, below). Some species showed little change in intensity between MALDI-1 and MALDI-2 (B) while many species dramatically increased with MALDI-2 (C-F). Values are listed of ion $\mathrm{m} / \mathrm{z}$, protein designation, amino acid sequence of peptide, and mass error. Assignments are based on accurate mass measurements matched to the in silico digest database within \pm 5 ppm error for

difference in distribution of average hydropathy values was observed (Figure S8). These results are consistent with the ionization mechanisms previously proposed for MALDI-2. It is thought that MALDI-2 proceeds through multiphoton ionization of matrix to form charged radicals and transfer charge to neutral analytes. ${ }^{19,30,31}$ This may result in a reversal of ion suppression effects for some analytes, previously demonstrated for analysis of lipids and certain classes of phospholipids with MALDI-2. ${ }^{23,32}$

Overall, IMS with MALDI-2 and subsequent tentative protein identification allowed for identification of $1,112 \pm 84$ unique peptides and $428 \pm 20$ unique proteins, $205 \pm 10$ of which were identified by 2 or more peptides, where $n=3$ in these experiments. In comparison, MALDI-1 led to approximately 3.5-fold fewer proteins

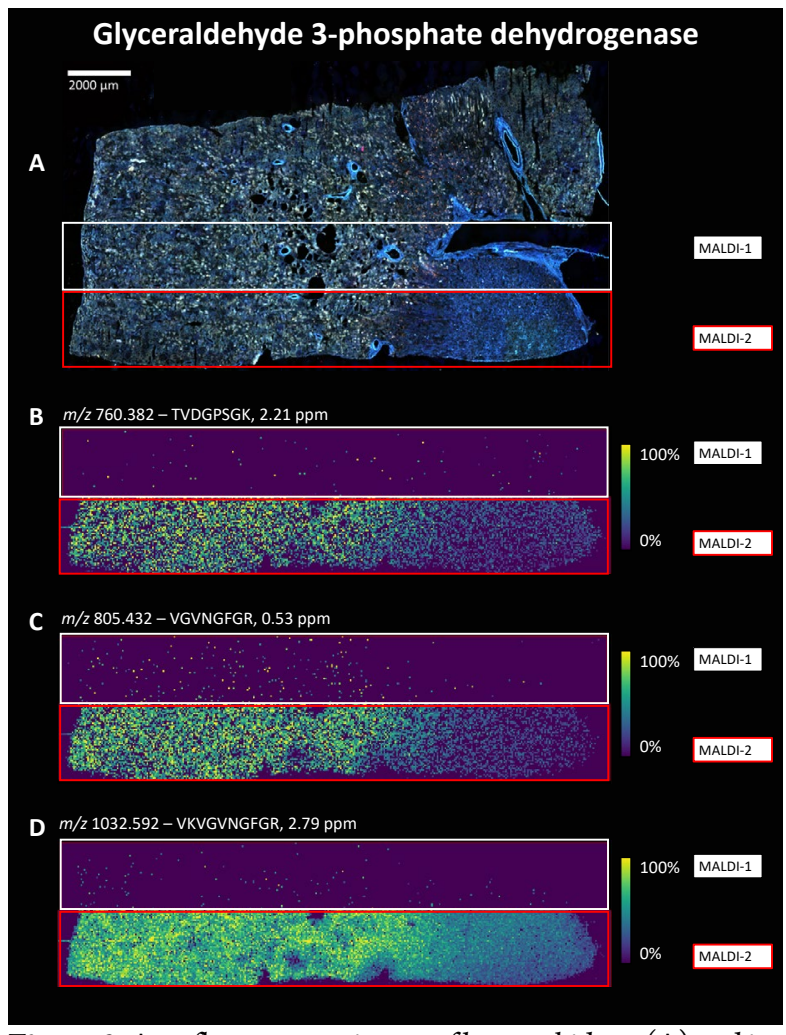

Figure 3. Autofluorescence image of human kidney (A) and ion images (B-D) showing multiple peptide matches to the protein glyceraldehyde 3-phosphate dehydrogenase. Amino acid sequence, measured $m / z$, and ppm error of each peptide is listed above the ion image. Three representative peptides are shown here, full data is displayed in Figure S8. Peptides localize similarly in the tissue which is expected from species relating to the nams:dant:find nuntrin

identified with two or more peptides (Table 1). In many cases, a protein was tentatively identified with MALDI- 2 only and these proteins may provide insight into biological functions and disease states. One example is glyceraldehyde 3-phosphate dehydrogenase, a protein that is overexpressed in kidney and liver cancer compared with healthy tissue. ${ }^{33}$ Here, the the peptides were shown to localize to the cortex of the kidney. The ion images of its corresponding peptides are shown in Figure 3 and Figure S9. Ion images for peptides are shown (Figure 3 B-D) where each peptide was contained in the theoretical peptide database and matched to the IMS data as described above. Examples of other clinically-significant proteins tentatively identified with MALDI-2 include alpha-enolase ${ }^{34}$, peroxiredoxin$1^{35}$, and phosphoglycerate kinase $1^{4}$, proteins that have been found to be elevated in some cancers and tumors.

Table 1. Number of unique proteins and peptides identified from thin tissue sections for MALDI- 1 and MALDI- $2(n=3)$. Identifications using the database generated from in silico digestion (A) allowed for significant increases versus the traditional approach comparing IMS data to LC-MS/MS results alone (B).

\begin{tabular}{|c|c|c|c|c|}
\hline & \multicolumn{2}{|c|}{ A) in silico digest } & \multicolumn{2}{|c|}{ B) LC-MS/MS only } \\
\hline & MALDI-1 & MALDI-2 & MALDI-1 & MALDI-2 \\
\hline Unique Peptides & $314 \pm 45$ & $1112 \pm 84$ & $39 \pm 9$ & $215 \pm 19$ \\
\hline Unique Proteins & s $197 \pm 19$ & $428 \pm 20$ & $17 \pm 1$ & $63 \pm 4$ \\
\hline de Proteins ( $\geq 2$ peptides) & $55 \pm 13$ & $205 \pm 10$ & $2 \pm 1$ & $43 \pm 3$ \\
\hline
\end{tabular}


These proof-of-concept experiments demonstrate the utility of MALDI-2 for enhanced detection of peptides from tissue sections for bottom-up protein IMS. This technology will be beneficial for future studies to obtain both protein identity and corresponding spatial distribution of clinically relevant peptide and protein species. These technical improvements are also critical for providing more robust and complete molecular imaging capabilities for large-scale tissue mapping projects such as the Human Biomolecular Atlas Program (HuBMAP), which will help provide a systems biology view on the molecular drivers of health and disease.

\section{ASSOCIATED CONTENT}

\section{Supporting Information}

Figure S1. Peptide ion images of 60 S ribosomal protein L18. Figure S2. Peptide ion images of Hemoglobin subunit alpha. Figure S3. Peptide ion images of Inactive phospholipase C-like protein 1. Figure S4. Peptide ion images of Glutathione S-transferase A2. Figure S5. Peptide ion images of Pyruvate Kinase PKM. Figure S6. Peptide ion images of Filamin B. Figure S7. Amino acid composition of identified peptides. Figure S8. Amino acid grand average of hydropathy (GRAVY) of peptides detected via MALDI-1 and MALDI-2. Figure S9. Peptide ion images corresponding to the protein identified as Glyceraldehyde 3-phosphate dehydrogenase. Sheet S1. IMS peak list matched to database of all theoretical peptides.

The Supporting Information is available free of charge on the ACS Publications website.

\section{AUTHOR INFORMATION}

\section{Author Contributions}

All authors have given approval to the final version of the manuscript.

Josiah C. McMillen: 0000-0002-1095-695X

Danielle B. Gutierrez: 0000-0001-6355-2134

Audra M. Judd: 0000-0002-4633-129X

Jeffrey M. Spraggins: 0000-0001-9198-5498

Richard M. Caprioli: 0000-0001-5859-3310

\section{ACKNOWLEDGMENT}

Support was provided by the NIH Common Fund and National Institute of Diabetes and Digestive and Kidney Diseases (U54DK120058 awarded to J.M.S. and R.M.C.), the NIH Common Fund and National Eye Institute (U54EY032442), and by the NIH National Institute of General Medical Sciences (2P41GM103391 awarded to R.M.C.). Human tissues were acquired through the Cooperative Human Tissue Network at Vanderbilt University Medical Center which is supported by the NIH National Cancer Institute (5 UM1 CA183727-08).

\section{REFERENCES}

Caprioli, R. M.; Farmer, T. B.; Gile, J. Molecular Imaging of Biological Samples: Localization of Peptides and Proteins Using MALDI-TOF MS. Anal. Chem. 1997, 69 (23), 4751-4760. https://doi.org/10.1021/AC970888I.

Altelaar, A. F. M.; Taban, I. M.; McDonnell, L. A.; Verhaert, P. D. E. M.; de Lange, R. P. J.; Adan, R. A. H.; Mooi, W. J.; Heeren, R. M. A.; Piersma, S. R. High-Resolution MALDI Imaging Mass Spectrometry Allows Localization of Peptide Distributions at Cellular Length Scales in Pituitary Tissue Sections. Int. J. Mass Spectrom. 2007, 260 (2-3), 203-211. https://doi.org/10.1016/j.ijms.2006.09.028.

(3) Wisztorski, M.; Desmons, A.; Quanico, J.; Fatou, B.; Gimeno, J.-P.; Franck, J.; Salzet, M.; Fournier, I. Spatially-Resolved Protein Surface Microsampling from Tissue Sections Using Liquid Extraction Surface Analysis. Proteomics 2016, 16 (11-12), 1622-1632. https://doi.org/10.1002/pmic.201500508.

Wang, J.; Ying, G.; Wang, J.; Jung, Y.; Lu, J.; Zhu, J.; Pienta, K. J.; Taichman, R. S. Characterization of Phosphoglycerate Kinase-1 Expression of Stromal Cells Derived from Tumor Microenvironment in Prostate Cancer Progression. Cancer Res. 2010, 70 (2), 471-480. https://doi.org/10.1158/0008-5472.CAN-09-2863.

(5) Ludwig, K.; Habbach, S.; Krieglstein, J.; Klumpp, S.; König, S. MALDI-TOF High Mass Calibration up to $200 \mathrm{KDa}$ Using Human Recombinant $16 \mathrm{KDa}$ Protein Histidine Phosphatase Aggregates.
PLoS One $\quad$ 2011,
https://doi.org/10.1371/journal.pone.0023612.

Gessel, M. M.; Norris, J. L.; Caprioli, R. M. MALDI Imaging Mass Spectrometry: Spatial Molecular Analysis to Enable a New Age of Discovery. J. Proteomics 2014, 107, 71-82. https://doi.org/10.1016/J.JPROT.2014.03.021.

(7) Prentice, B. M.; Ryan, D. J.; Van de Plas, R.; Caprioli, R. M.; Spraggins, J. M. Enhanced Ion Transmission Efficiency up to m/z 24000 for MALDI Protein Imaging Mass Spectrometry. Anal. Chem. 2018, 90 (8), 5090-5099. https://doi.org/10.1021/acs.analchem.7b05105.

Piga, I.; Heijs, B.; Nicolardi, S.; Giusti, L.; Marselli, L.; Marchetti, P.; Mazzoni, M. R.; Lucacchini, A.; McDonnell, L. A. Ultra-High Resolution MALDI-FTICR-MSI Analysis of Intact Proteins in Mouse and Human Pancreas Tissue. Int. J. Mass Spectrom. 2019, 437, 10-16. https://doi.org/10.1016/j.ijms.2017.11.001.

(9) Minerva, L.; Boonen, K.; Menschaert, G.; Landuyt, B.; Baggerman, G.; Arckens, L. Linking Mass Spectrometric Imaging and Traditional Peptidomics: A Validation in the Obese Mouse Model. Anal. Chem. 2011, 83 (20), 7682-7691. https://doi.org/10.1021/ac200888j.

(10) Gustafsson, J. O. R.; Oehler, M. K.; McColl, S. R.; Hoffmann, P. Citric Acid Antigen Retrieval (CAAR) for Tryptic Peptide Imaging Directly on Archived Formalin-Fixed Paraffin-Embedded Tissue. J. Proteome 
Res. 2010, 9 (9), 4315-4328. https://doi.org/10.1021/pr9011766.

$(11)$

Judd, A. M.; Gutierrez, D. B.; Moore, J. L.; Patterson, N. H.; Yang, J.; Romer, C. E.; Norris, J. L.; Caprioli, R. M. A Recommended and Verified Procedure for in Situ Tryptic Digestion of Formalin-Fixed Paraffin-Embedded Tissues for Analysis by Matrix-Assisted Laser Desorption/Ionization Imaging Mass Spectrometry. J. Mass Spectrom. 2019, 54 (8), 716-727. https://doi.org/10.1002/jms.4384.

Heijs, B.; Carreira, R. J.; Tolner, E. A.; De Ru, A. H.; Van Den Maagdenberg, A. M. J. M.; Van Veelen, P. A.; McDonnell, L. A. Comprehensive Analysis of the Mouse Brain Proteome Sampled in Mass Spectrometry Imaging. Anal. Chem. 2015, 87 (3), 1867-1875. https://doi.org/10.1021/ac503952q.

(13) Crank, J. A.; Armstrong, D. W. Towards a Second Generation of Ionic Liquid Matrices (ILMs) for MALDI-MS of Peptides, Proteins, and Carbohydrates. J. Am. Soc. Mass Spectrom. 2009, 20 (10), 1790-1800. https://doi.org/10.1016/j.jasms.2009.05.020.

(14) Thomas, A.; Patterson, N. H.; Laveaux Charbonneau, J.; Chaurand, P. Orthogonal Organic and Aqueous-Based Washes of Tissue Sections to Enhance Protein Sensitivity by MALDI Imaging Mass Spectrometry. J. Mass Spectrom. 2013, 48 (1), 42-48. https://doi.org/10.1002/jms.3114.

(15) Cillero-Pastor, B.; Heeren, R. M. A. Matrix-Assisted Laser Desorption Ionization Mass Spectrometry Imaging for Peptide and Protein Analyses: A Critical Review of On-Tissue Digestion. 2013. https://doi.org/10.1021/pr400743a.

(16) Angel, P. M.; Mehta, A.; Norris-Caneda, K.; Drake, R. R. MALDI Imaging Mass Spectrometry of N-Glycans and Tryptic Peptides from the Same Formalin-Fixed, Paraffin-Embedded Tissue Section. In Methods in Molecular Biology; Sarwal, M. M., Sigdel, T. K., Eds.; New York, NY, 2018; Vol. 1788, pp 225-241. https://doi.org/10.1007/7651_2017_81.

(17) Prentice, B. M.; Ryan, D. J.; Grove, K. J.; Cornett, D. S.; Caprioli, R. M.; Spraggins, J. M. Dynamic Range Expansion by Gas-Phase Ion Fractionation and Enrichment for Imaging Mass Spectrometry. Anal. Chem. 2020, $92 \quad$ (19), 13092-13100 https://doi.org/10.1021/acs.analchem.0c02121.

(18) Soltwisch, J.; Kettling, H.; Vens-Cappell, S.; Wiegelmann, M.; Müthing, J.; Dreisewerd, K. Mass Spectrometry Imaging with LaserInduced Postionization. Science (80-. ). 2015, 348 (6231), 211-215. https://doi.org/10.1021/cr010375i.

(19) Soltwisch, J.; Heijs, B.; Koch, A.; Vens-Cappell, S.; Ho, J.; Dreisewerd, K. MALDI-2 on a Trapped Ion Mobility Quadrupole Time-of-Flight Instrument for Rapid Mass Spectrometry Imaging and Ion Mobility Separation of Complex Lipid Profiles. 2020, 06, 15. https://doi.org/10.1021/acs.analchem.0c01747.

(20) Barré, F. P. Y.; Paine, M. R. L.; Flinders, B.; Trevitt, A. J.; Kelly, P. D.; Ait-Belkacem, R.; Garcia, J. P.; Creemers, L. B.; Stauber, J.; Vreeken, R. J.; et al. Enhanced Sensitivity Using Maldi Imaging Coupled with Laser Postionization (Maldi-2) for Pharmaceutical Research. Anal. Chem. 2019, 91 (16), 10840-10848. https://doi.org/10.1021/acs.analchem.9b02495.

Heijs, B.; Potthoff, A.; Soltwisch, J.; Dreisewerd, K. MALDI-2 for the Enhanced Analysis of N -Linked Glycans by Mass Spectrometry
Imaging . Anal. Chem. 2020, 92 (20), 13904-13911. https://doi.org/10.1021/acs.analchem.0c02732.

(22) Woods, A. S.; Jackson, S. N.; Lewis, E. K.; Egan, T.; Muller, L.; Tabet, J. C.; Schultz, J. A. MALDI/Post Ionization-Ion Mobility Mass Spectrometry of Noncovalent Complexes of Dopamine Receptors' Epitopes. J. Proteome Res. 2013, 12 (4), 1668-1677. https://doi.org/10.1021/pr301004w.

McMillen, J. C.; Fincher, J. A.; Klein, D. R.; Spraggins, J. M.; Caprioli, R. M. Effect of MALDI Matrices on Lipid Analyses of Biological Tissues Using MALDI-2 Post-Ionization Mass Spectrometry. J. Mass Spectrom. https://doi.org/10.1002/JMS.4663.

Gutierrez, D.; Allen, J.; Jenkins, Z.; Spraggins, J. LC-MS/MS Label-Free Proteomic Data Acquisition V.3; 2020. https://doi.org/10.17504/protocols.io.bft3jnqn.

Allen, J.; Spraggins, J.; Gutierrez, D. Automated, Rapid Preparation of Tissue Sections for Proteomic Analysis V.2; 2019. https://doi.org/10.17504/protocols.io.67nhhme.

Thiede, B.; Höhenwarter, W.; Krah, A.; Mattow, J.; Schmid, M.; Schmidt, F.; Jungblut, P. R. Peptide Mass Fingerprinting. Methods 2005, $35 \quad$ (3 SPEC.ISS.), 237-247. https://doi.org/10.1016/j.ymeth.2004.08.015.

Ellis, S. R.; Soltwisch, J.; Paine, M. R. L.; Dreisewerd, K.; Heeren, R. M. A. Laser Post-Ionisation Combined with a High Resolving Power Orbitrap Mass Spectrometer for Enhanced MALDI-MS Imaging of Lipids. Chem. Commun. 2017, 53 (53), 7246-7249. https://doi.org/10.1039/C7CC02325A.

(28) Kyte, J.; Doolittle, R. F. A Simple Method for Displaying the Hydropathic Character of a Protein. J. Mol. Biol. 1982, 157 (1), 105132. https://doi.org/10.1016/0022-2836(82)90515-0.

Maria Nadler, W.; Waidelich, D.; Kerner, A.; Hanke, S.; Berg, R.; Trumpp, A.; Ro, C. MALDI versus ESI: The Impact of the Ion Source on Peptide Identification. J. Proteome Res. 2017, 16, 1207-1215. https://doi.org/10.1021/acs.jproteome.6b00805.

Hanley, L.; Wickramasinghe, R.; Yung, Y. P. Laser Desorption Combined with Laser Postionization for Mass Spectrometry. Annu. Rev. Anal. Chem. 2019, 12 (1), annurev-anchem-061318-115447. https://doi.org/10.1146/annurev-anchem-061318-115447.

Potthoff, A.; Dreisewerd, K.; Soltwisch, J. Detailed Characterization of the Postionization Efficiencies in MALDI-2 as a Function of Relevant Input Parameters. J. Am. Soc. Mass Spectrom. 2020, 31 (9), 1844-1853. https://doi.org/10.1021/jasms.0c00072.

Boskamp, M. S.; Soltwisch, J. Charge Distribution between Different Classes of Glycerophospolipids in MALDI-MS Imaging. Anal. Chem. 2020. https://doi.org/10.1021/acs.analchem.9b05761.

(33) Tarrado-Castellarnau, M.; Diaz-Moralli, S.; Polat, I. H.; SanzPamplona, R.; Alenda, C.; Moreno, V.; Castells, A.; Cascante, M. Glyceraldehyde-3-Phosphate Dehydrogenase Is Overexpressed in Colorectal Cancer Onset. Transl. Med. Commun. 2017, 2 (1), 6. https://doi.org/10.1186/s41231-017-0015-7.

Gao, J.; Zhao, R.; Xue, Y.; Niu, Z.; Cui, K.; Yu, F.; Zhang, B.; Li, S. Role of Enolase-1 in Response to Hypoxia in Breast Cancer: Exploring the 
Mechanisms of Action. Oncol. Rep. 2013, 29 (4), 1322-1332. https://doi.org/10.3892/or.2013.2269.

(35) Cai, C. Y.; Zhai, L. L.; Wu, Y.; Tang, Z. G. Expression and Clinical Value of Peroxiredoxin-1 in Patients with Pancreatic Cancer. Eur. J. Surg. Oncol. 2015, 41 (2), 228-235. https://doi.org/10.1016/j.ejso.2014.11.037. 
For Table of Contents Only:

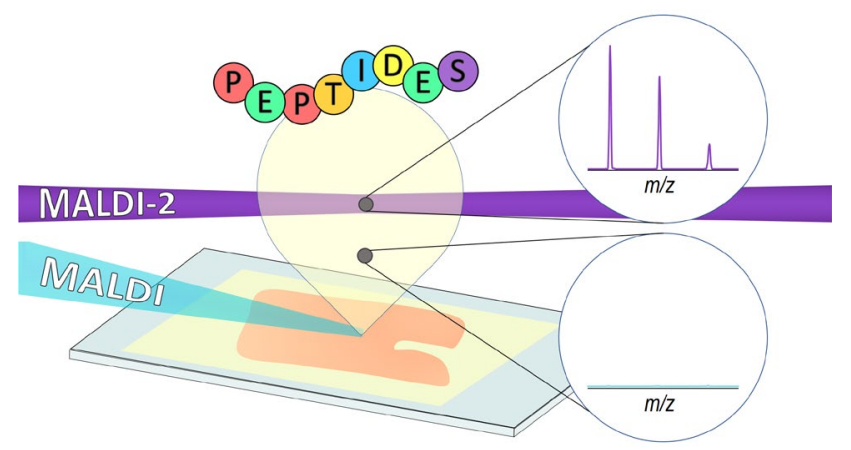

